\title{
A method to measure redox potential (Eh) and pH in agar media and plants shows that fungal growth is affected by and affects $\mathrm{pH}$ and $\mathrm{Eh}$
}

Lydia Bousset $^{\text {a }}$, Magali Ermel ${ }^{\text {a }}$, Brigitte Soglonou ${ }^{\text {b }}$, Olivier Husson ${ }^{\text {b c, d }}$

a INRA, UMR1349 IGEPP, France

${ }^{b}$ Africa Rice Center, 01 BP 2551, Bouaké, 01, Cote d'Ivoire

${ }^{c}$ CIRAD, UPR AIDA, F-34398 Montpellier, France

${ }^{d}$ AIDA, Univ. Montpellier, CIRAD, Montpellier, France

\begin{abstract}
The specificities of the plant environment and its effects on fungal growth are not yet fully explored. Both $\mathrm{pH}$ and Eh play a key role during this interaction, but are often studied independently or at different scales. We aimed at investigating whether the methods developed for the joint characterization of the $\mathrm{pH}$ and Eh in soil could be transposed to fungi. On artificial media, the growth of all 16 species tested significantly altered either $\mathrm{Eh}, \mathrm{pH}$ or both. Measuring Eh reveals that even the species not modifying $\mathrm{pH}$ can have an impact on the surrounding environment. Reciprocally, fungi responded to $\mathrm{pH}$ and Eh parameters, both quantitatively with a decrease in colony diameter and qualitatively with colony aspect repeatedly and thoroughly modified. In infected oilseed rape plant stems, $\mathrm{pH}$ and Eh were significantly altered. The observed alcalinisation or acidification correlates with canker length. The joint characterization of both parameters will allow understanding the impact of fungi on their environment, and conversely of the environment on fungal growth. The availability of methods for measurement opens the prospect to study combinations of stresses, and get an understanding of the involvement of $\mathrm{pH}$ and $\mathrm{Eh}$ modifications in these interactions.
\end{abstract}

Keywords: Agricultural microbiology, Leptosphaeria maculans, Measurement, Oxido-reduction, Plant pathogen, Sclerotinia sclerotiorum

\section{Highlights}

- The procedure for soil can be extended for measurement in agar media and infected plants.

- The growth of fungi tested significantly altered either Eh, $\mathrm{pH}$ or both in agar media.

- Even the species not modifying $\mathrm{pH}$ can alter Eh in the surrounding environment.

- Reciprocally, on altered media fungal growth was modified.

- In plant stems, alkalinization or acidification correlates with canker length.

\section{Introduction}

Fungal plant pathogens need to get resources from the plant and for doing so they need to live in the specific environment of plants (Braunsdorf et al., 2016, Zeilinger et al., 2016, van der Does and Rep, 2017). Getting a better understanding of the interaction between pathogens and their host plants might open new ways to control plant diseases. Yet, the specificities of the plant environment and its effects on fungal growth are not yet fully explored further than the molecular dialog between partners (Lo Presti et al., 2015, Jwa and Hwang, 2017). To decipher the interrelation between plant growth and their soil environment, recent theoretical and methodological advances have been made through the joint characterization of the $\mathrm{pH}$ and Eh (Husson, 2013, Husson et al., 2018). This opens the prospect to develop similar methods for fungi, because both $\mathrm{pH}$ and Eh are important in their metabolism.

As compared to other microorganisms, fungi are capable of growth and development over wide $\mathrm{pH}$ ranges. Several species, including plant pathogens, can actively modulate the $\mathrm{pH}$ of their environment by secreting acids or alkali (Landraud et al., 2013, Vylkova, 2017). The ability to control extracellular $\mathrm{pH}$ is an important aspect of fungal physiology that contributes to fitness within the host (Vylkova, 2017). $\mathrm{pH}$ matters for the interaction between the fungus and its host plants, from field to molecular scales. At the field scale, opposite effect can be observed for wheat soilborne diseases: take-all (Gaeumannomyces graminis var. tritici) is more severe in alkaline soils (Smiley, 1974, Lebreton et al., 2014) and cephalosporium stripe 
(Cephalosporium gramineum) severity increase with soil acidity (Stiles and Murray, 1996). At the molecular level, $\mathrm{pH}$ alters gene expression (Daval et al., 2013). Thus for fungi, $\mathrm{pH}$ is an important characteristic of the environment. It has however been stressed that because of their interrelation, analyses of $\mathrm{pH}$ gain to be associated with measures of Eh, that is oxido-reduction status (Husson et al., 2016, Husson et al., 2018).

So far, in plant-pathogenic fungi interactions, oxido-reduction processes are documented mostly at cellular and molecular levels (Lehmann et al., 2015, Jwa and Hwang, 2017). Plants have been investigated more thoroughly. Oxido-reduction reactions and reactive oxygen species have a profound influence on almost every aspect of plant biology especially to balance information from metabolism and the environment (Dietz et al., 2016, Foyer and Noctor, 2016), with reactive oxygen species (ROS) and reactive nitrogen species (RNS) as key signaling molecules that facilitate a plethora of adaptive metabolic, molecular genetic and epigenetic responses (Foyer and Noctor, 2005, Foyer and Noctor, 2016). As oxido-reduction processes play a major role in plant biology, developing methods to investigate if this occurs also in fungi is desirable.

Eh and $\mathrm{pH}$ are interacting, as oxidation leads to acidification, and the importance of measuring both parameters has been documented on soils (Husson et al., 2018). Eh and pH regulation is central in plant physiology and phenology and as the vast majority of biotic and abiotic stresses are translated into redox signals in plants (Husson, 2013). The leaf $\mathrm{Eh}$ and $\mathrm{pH}$ were proposed in previous studies to explain the variable resistance of cereals to obligates parasites (Benada, 2017). Investigating these topics further could benefit from the recent methodological developments. The aim of our study was to investigate whether the methods developed for soil (Husson et al., 2016, Husson et al., 2018) could be transposed to fungi. We first worked on artificial media, assessing the impact of fungal growth on the media in fungal cultures. Reciprocally, altered media were used to characterize sensitivity of fungal growth to both $\mathrm{pH}$ and Eh parameters. Later, we tested how the measurement can be performed with fungi growing on oilseed rape plants, as a first step towards the same studies in conditions matching the natural environment of fungal pathogens.

\section{Materials and methods}

\subsection{Culture media}

To test how fungal growth modified the medium on which they grew, four independent experiments were performed with 6 fungal strains (Table 1). In the Mar2016 experiment, 13 additional strains were included (Table 1). All tests were performed on malt-agar media (Malt extract $20 \mathrm{~g} \mathrm{l}^{-1}$, Agar $20 \mathrm{~g} \mathrm{l}^{-1}$, Streptomycin $0.1 \mathrm{~g} \mathrm{l}^{-1}$ ). All culture media were autoclaved for $20 \mathrm{mn}$ at $120{ }^{\circ} \mathrm{C}$ and cooled down before Streptomycin concentrated solution $10 \% \mathrm{w} / \mathrm{v}$ in water was added. Within each experiment, replicates were prepared independently and corresponding uninoculated aliquots were kept for $\mathrm{pH}$ and Eh initial and final measurements (Table 1). Fungal cultures were grown in sterile $6 \mathrm{~cm}$ diameter plastic containers $(50 \mathrm{ml})$ commonly used to store and reheat food sauce. 
Table 1. List and origin of 19 fungal strains used in the 4 experiments (Jan2015 to Mar2016) with dates of inoculation, final measurements for media and fungal growth. "nd" stands for unknown.

\begin{tabular}{|c|c|c|c|c|c|c|c|c|c|c|}
\hline Fungus (Genus species) & Jan2015 & Feb2015 & Jul2015 & Mar2016 & Name & Other name & Year & Host plant & Country & Provided by \\
\hline Inoculation & $13 / 01 / 2015$ & $27 / 02 / 2015$ & $27 / 04 / 2015$ & $26 / 02 / 2016$ & & & & & & \\
\hline Media final measurements & $30 / 01 / 2015$ & $16 / 03 / 2015$ & $10 / 08 / 2015$ & as below & & & & & & \\
\hline & & & & & & & & & & \\
\hline \multicolumn{11}{|c|}{ Fungal growth final measurements: } \\
\hline Rhizoctonia solani & & & & $04 / 03 / 2016$ & RsAG21 & R5 & nd & radish & UK & D. Bailey \\
\hline Rhizoctonia solani & & & & $04 / 03 / 2016$ & RsAG22 & RG6 $n^{\circ} 9$ & nd & beetroot & nd & K. Bouchek \\
\hline Rhizoctonia solani & & & & $04 / 03 / 2016$ & RsAG3 & $\mathrm{RH} 1 n^{\circ} 6$ & 2010 & potato & France & K. Bouchek \\
\hline Sclerotinia sclerotiorum & $15 / 01 / 2015$ & $02 / 03 / 2015$ & $27 / 07 / 2015$ & $04 / 03 / 2016$ & Ss448 & Scl 448 & 1987 & oilseed rape & Canada & H. Brun \\
\hline Sclerotinia minor & $15 / 01 / 2015$ & $02 / 03 / 2015$ & $27 / 07 / 2015$ & 04/03/2016 & Sm713 & Scl 713 & 1989 & letuce & France & Grisp \\
\hline Sclerotinia trifoliorum & & & & $04 / 03 / 2016$ & St535 & Scl535 & 1973 & alfalfa & France & G Raynal \\
\hline Alternaria brassicicola & $16 / 01 / 2015$ & $03 / 03 / 2015$ & $28 / 07 / 2015$ & $11 / 03 / 2016$ & Ab177 & Alt 177 & 1982 & mustard & France & H. Brun \\
\hline Botrytis cinerea & & & & $11 / 03 / 2016$ & Bc1 & Bc1 & 2016 & oilseed rape & France & L. Bousset \\
\hline Penicillium sp. & & & & $11 / 03 / 2016$ & Ps1 & $\mathrm{Bc} 258$ & nd & nd & France & H. Brun \\
\hline Fusarium roseum & & & & $11 / 03 / 2016$ & Fr728 & 728 & 1990 & oilseed rape & France & H. Brun \\
\hline Magnaporthe oryzae & & & & $11 / 03 / 2016$ & Mo13 & Fr0013 & 1988 & rice & France & D. Tharreau \\
\hline Leptosphaeria biglobosa & $23 / 01 / 2015$ & $16 / 03 / 2015$ & $10 / 08 / 2015$ & $18 / 03 / 2016$ & LbB3.5 & B3.5 & 1996 & mustard & France & H. Brun \\
\hline Leptosphaeria maculans & $29 / 01 / 2015$ & $16 / 03 / 2015$ & $10 / 08 / 2015$ & $18 / 03 / 2016$ & LmJN2 & JN2 & 2001 & nd & in vitro crosses & MH. Balesdent \\
\hline Leptosphaeria maculans & & & & $18 / 03 / 2016$ & LmNzT4 & NzT4 & nd & nd & New Zealand & MH. Balesdent \\
\hline Mycosphaerella brassicicola & & & & $18 / 03 / 2016$ & Mb338 & 338 & 1986 & cabbage & France & H. Brun \\
\hline Verticillium dahliae & $23 / 01 / 2015$ & 09/03/2015 & 03/08/2015 & $18 / 03 / 2016$ & Vd586 & V 586 & 1988 & nd & Germany & V. Paul \\
\hline Verticillium longisporum & & & & $18 / 03 / 2016$ & VI668 & V 668 & 1989 & oilseed rape & France & H. Brun \\
\hline Pyrenopezziza brassicae & & & & $04 / 04 / 2016$ & $\mathrm{~Pb} 1$ & $\mathrm{~Pb} 1$ & 2016 & oilseed rape & France & L. Bousset \\
\hline Pseudocercosporella capsellae & & & & $04 / 04 / 2016$ & Pc1 & Pc1 & 2015 & oilseed rape & France & L. Bousset \\
\hline
\end{tabular}

To test how modified $\mathrm{pH}$ and Eh media altered fungal growth, within each of the 3 experiments (Jan2015, Feb2015, Ju12015) three replicates of a range of 13 malt-agar media (Malt extract $20 \mathrm{~g} \mathrm{l}^{-1}$, Agar $20 \mathrm{~g} \mathrm{l}^{-1}$, Streptomycin $0.1 \mathrm{~g} \mathrm{l}^{-1}$ ) were prepared independently. To alter $\mathrm{Eh}$ and $\mathrm{pH}$ without changing the composition of the media, we used electrolyzed water products, because when chemical compounds are added, it is difficult to disentangle the effects via $\mathrm{pH}$ from directs effects (Bekker et al., 2009). The range of agar media included native one (N0) and four sets of three media with part of the water (in proportions $1: 3 ; 1: 1$ and

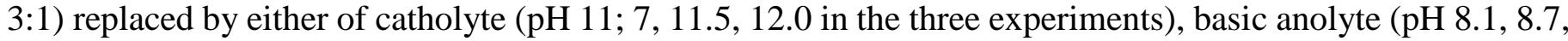

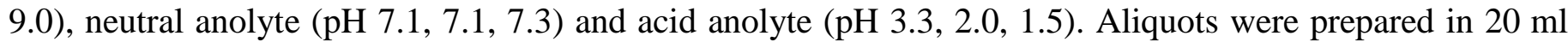
flacons for uninoculated measurements at the start and end of the experiment. Aliquots of $10 \mathrm{ml}$ in $5 \mathrm{~cm}$ diameter Petri dishes were prepared for inoculation and fungal growth measurement. For each experiment, anolyte and catholyte were obtained from IDEEAQUACULTURE, Parc Euromédecine 2, 39 Rue Jean Giroux, 34080 Montpellier, France. Products were freshly prepared 24-48 h before media preparation, using Envirolyte ELA900 and shipped in light-opaque containers. Eh and $\mathrm{pH}$ of the products were measured on aliquots before use. These products are all obtained from water, and the only compound added during the production is $\mathrm{NaCl}$. We thus made a specific experiment (Sep2015, see below) to check that within the range of final $\mathrm{NaCl}$ concentrations in the media, fungal growth was not altered for any of the 6 fungal species (Fig. S1).

To test how modified $\mathrm{pH}$ and Eh media altered fungal growth of Magnaporthe oryzae, within two experiments (Jan2016, Mar2016) four replicates of a range of 13 rice flour-agar media (Rice flour $10 \mathrm{~g} \mathrm{l}^{-1}$, Agar $15 \mathrm{~g} \mathrm{l}^{-1}$, Yeast extract $2 \mathrm{~g} \mathrm{l}^{-1}$, Penicillin $0.1 \mathrm{~g} \mathrm{l}^{-1}$ ) were prepared. The range of rice flour agar media included native one (N0), addition of Hydrogen peroxide, addition of Hydroquinone and the combination of both. This made 20 media in Jan 2016 with combinations (Hydroquinone-Hydrogen peroxide) 0-0; 0-0.05; $0-0.1 ; 0-0.2 ; 0-0.4 ; 0-0.8 ; 0-1.6 ; 4-0 ; 4-0.1 ; 16-0 ; 16-0.05 ; 16-0.1 ; 16-0.2 ; 16-0.4 ; 64-0 ; 64-0.1 ; 256-0$; $256-0.05 ; 256-0.1 ; 256-0.4$ and 14 media in Mar2016 with combinations (Hydroquinone-Hydrogen peroxide) $0-0 ; 0-1 ; 0-2 ; 0-4 ; 50-0 ; 50-2 ; 100-0 ; 100-1 ; 100-2 ; 100-4 ; 20-0 ; 200-2 ; 300-0 ; 300-4$.

\subsection{Fungal growth experiments}

A total of 19 isolates of 16 fungal species were used (Table 1). For each isolate, $5 \mathrm{~mm}$ diameter agar plugs of fresh cultures on malt-agar were prepared, placed in the center of Petri dishes and fungal growth was measured 
as two perpendicular diameters of the colony (Table 1). To assess the impact of fungal growth on the medium, plastic containers were used instead of Petri dishes in order to allow sufficient depth for the electrodes after colony growth. Agar plug was placed in the center, and final measurements were performed by sticking electrodes through the colony, on both sides of the plug. In each of the 3 replicates, there was 1 Petri dish and 1 container in Jan2015 and Feb2015; 2 Petri dishes in Sep2015; 3 Petri dishes and 3 containers in Jul2015 and 3 containers in Mar2016 experiments, respectively. All cultures and uninoculated aliquots were kept at room temperature in INRA Le Rheu, with no supplementary light. Hourly temperature (mean $21.7^{\circ} \mathrm{C}$ se 1.6 ) and humidity (mean $46.5 \%$ se 8.9 ) were recorded with a TEMPCO Hygro bouton 23 data logger. M. oryzae growth experiments were performed at Africarice, Cotonou, Bénin with local strains BN1162 in Jan16 and BN1168 in Mar16 on rice flour-agar.

\subsection{Oxido-reduction potential (ORP) electrodes and voltmeter}

Redox potential was measured in agar media as Described by Husson et al. (2016) using Ag/AgCl Reference electrode Radiometer analytical E21M002 and Radiometer Analytical Pt plate electrode $5 \times 5 \mathrm{~mm} \mathrm{M} 241 \mathrm{Pt}$, with a Voltcraft VC850 multimeter $\left(10 \times 10^{6} \mathrm{Ohm}\right.$ input resistance) for all fungi and a WTW 3110 $\left(10 \times 10^{12} \Omega\right.$ input resistance) for $M$. oryzae growth experiments. The Eh value considered was the Eh (in $\mathrm{mV}$ ) measured according to $\mathrm{Ag} / \mathrm{AgCl}$ reference electrode, then transformed to give Eh according to the Normal Hydrogen Electrode (ENH). $\mathrm{pH}$ was measured in agar media with a with a Voltcraft VC850 multimeter $\left(10 \times 10^{6} \mathrm{Ohm}\right.$ input resistance) using $\mathrm{Ag} / \mathrm{AgCl}$ reference electrode Radiometer analytical E21M002 and a glass $\mathrm{pH}$ electrode Radiometer analytical pHG301-9. Data were converted into $\mathrm{pH}$ values using linear regression of the measured values of standard solutions.

\subsection{Plants experiments}

Winter oilseed rape was sown in 03/10/2017 in $12 \times 12 \times 21 \mathrm{~cm}$ pots with a 1:1:1 mix of sand, peat and compost and grown outside at INRA Le Rheu $\left(48 \cdot 1^{\circ} \mathrm{N}, 1 \cdot 5^{\circ} \mathrm{W}\right)$, in Brittany, France. There was three replicates of 38 plants for Leptosphaeria maculans experiments and 2 replicates of 38 and 44 plants for Sclerotinia sclerotiorum experiments.

Natural infections with L. maculans airborne ascospores occurred on some of the plants, with typical leaf spots observed in November and cankers at flowering. Measurements were performed on infected and uninfected plants after flowering, over $4 \mathrm{~d}$ (02/05; 07/05; 22/05, 28/05). Plant stem was cut lengthwise, and internal canker length was recorded. Immediately after, a $2 \mathrm{~cm}$ portion of the stem taken at crown level was cut and quickly grinded with a mortar and pestle. The plant material was then placed in the barrel of a $2 \mathrm{ml}$ syringe from which the nozzle had been cut. For the redox potential measurement with the Voltcraft VC850 multimeter, we used a $9 \mathrm{~cm}$ Petri dish with a filter paper moistened with $0.1 \mathrm{M} \mathrm{KCl}$ solution (Fig. S2). The $\mathrm{Ag} / \mathrm{AgCl}$ reference electrode was placed standing, with tip touching the moist filter paper. The Pt plate electrode was placed in the plant material in the syringe, then the syringe barrel was placed standing with tip touching the moist filter paper. After reading the redox potential, the plunger of the syringe was placed in the barrel to squeeze the liquid onto a Horiba LAQUAtwin-pH-22 $\mathrm{m}$ for $\mathrm{pH}$ measurement.

Inoculations with $S$. sclerotiorum were performed at the time of petal fall. Matchsticks pieces were autoclaved in liquid $2 \%$ malt medium. Agar plugs from fresh culture were transferred (13/04 and 30/04) to the center of $9 \mathrm{~cm}$ diameter malt-agar Petri dishes with lying matchsticks pieces, and cultured at $20{ }^{\circ} \mathrm{C}$. On 17/04 and 03/05 for each of the 2 plant replicates, a matchstick piece covered with mycelium was tightly fitted through the $3 \mathrm{~mm}$ diameter hole drilled at $10 \mathrm{~cm}$ height through the stem of 16 and 24 inoculated and 19 and 23 mock-inoculated plants. External canker length was measured 20/04 and 07/05, immediately after, a $2 \mathrm{~cm}$ portion of the stem taken at the inoculation point was cut and processed for Eh and $\mathrm{pH}$ measurement as described above.

\subsection{Data analysis}

Statistical analyses on artificial media experiment data were performed using XLSTAT (Addinsoft, 2016). Analysis of variance (ANOVA) was conducted on Eh, $\mathrm{pH}$ for comparison of agar media after fungal growth. ANOVAs were followed by a comparison of means using the Ryan-Einot-Gabriel-Welsh test Q (REGW-Q) as it provides the best compromise between the need to have a powerful test and the need to limit the 
familywise error rate at $\alpha$, according to Howell (2009). Quadratic regressions between colony growth, $\mathrm{pH}$ and Eh were performed for the different 7 fungal species on altered agar media.

Statistical analyses on plant experiment data were performed using R software (R Core Team, 2013). Generalized linear modeling was used to investigate the effects of inoculation, canker on $\mathrm{pH}$ measured. For plant $\mathrm{pH}$ and $\mathrm{Eh}$ data, we used a Gaussian linear model on log-transformed data. Linear contrasts were calculated with as Estimated Marginal Means with emmeans package to determine specific differences among treatment combinations.

\section{Results}

\subsection{Effect of fungi on $\mathrm{pH}$ and Eh in the agar media}

Uninoculated malt-agar media were acid (pH from 4.5 to 5) and oxidized (Eh from 500 to 600) (Fig. 1). For all tested species, ANOVA analyses followed by REGW-Q comparison of means indicated that fungal growth significantly altered $\mathrm{pH}$ and Eh in agar media (Fig. 1A, B; Table 2). These alterations were species-specific and in directions opposite to the slight evolution of uninoculated media between initial (empty symbols) and final (filled symbols) measurements. All Sclerotinia species acidified the media, Sclerotinia minor and S. sclerotiorum did not change Eh, while Sclerotinia trifolorium also reduced the media (Fig. 1A). M. oryzae, Penicillium sp. and Pyrenopezziza brassicae alkalinized the media, with variable intensities of reduction. The main effect of other species was reduction, either mild (Bc1, Mb, Pc) intermediate ( $\mathrm{Lm}, \mathrm{Vd})$ or intense (Rs, $\mathrm{Ab}, \mathrm{Lb}, \mathrm{Fr}$ ). Results of independent experiments with 6 species were congruent (Fig. 1B). The Eh-pH couples obtained after the growth of the fungal species tested did not align on the theoretical correlation between Eh and $\mathrm{pH}$, with a negative slope of $59 \mathrm{mV}$ decrease in Eh for one unit increase in $\mathrm{pH}$ at $25^{\circ} \mathrm{C}$, according to the equation of Nernst (Fig. 1A, B).

Fig. 1. Effect of fungal growth on the $\mathrm{pH}$ and Eh of the malt-agar media. (A) Following the growth of 19 fungi in March16 experiment; (B) Following the growth of 6 fungi in 4 independent experiments of Jan2015 (diamonds) Feb2015 (triangles) Jul2015 (squares) and Mar2016 (circles) with overall means (crosses). Symbols in Panel A correspond to measurement at different final dates (see Table 1). In panel B uninoculated medium (black) was measured at the start ( $N i$, empty symbols) and at the end (Ni_F, filled symbols) of the experiments. Plain line shows the theoretical relationship between $\mathrm{pH}$ and Eh according to the equation of Nernst. Dashed lines correspond to significantly different groups for $\mathrm{pH}$ (horizontal) and Eh (vertical) in the $R E G W-Q$ analysis after ANOVA (see Table 2).
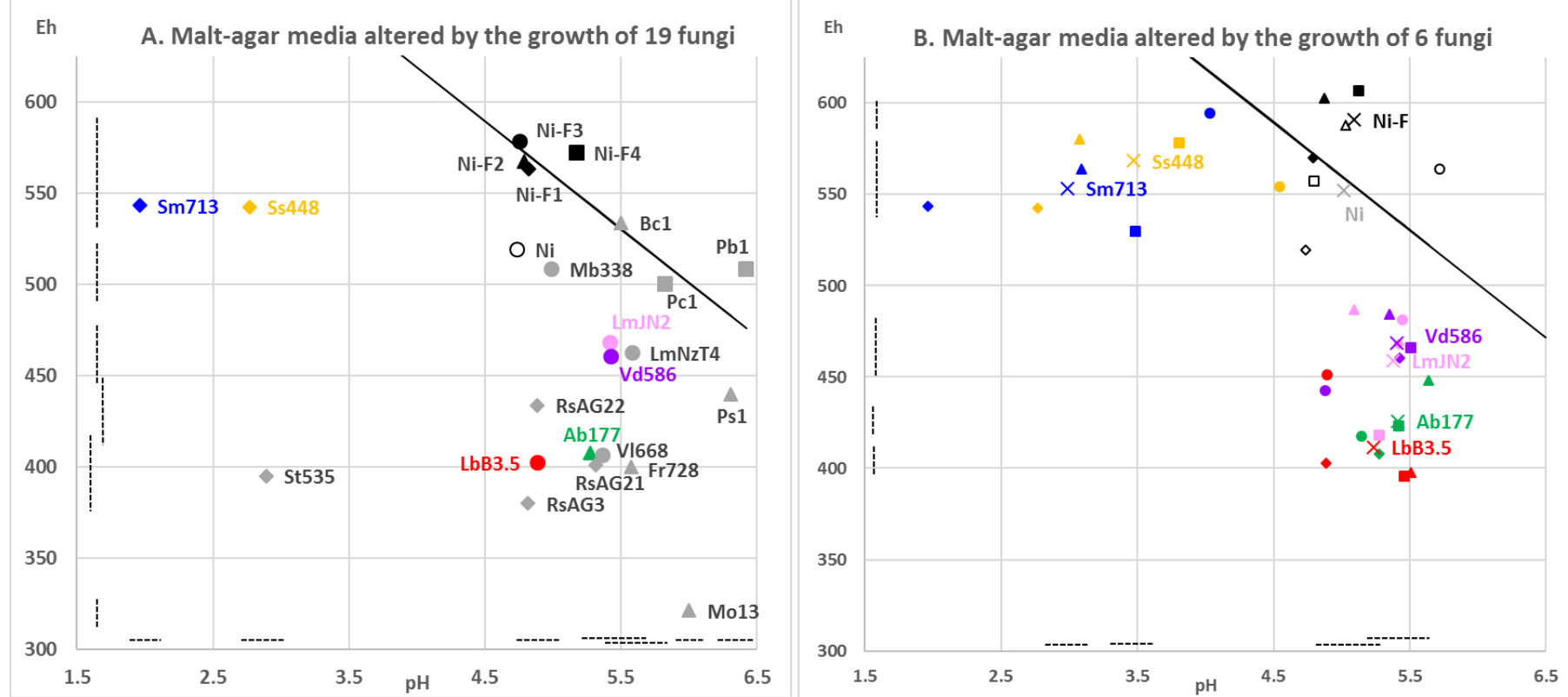
Table 2. For each figure and each experiment, mean and standard error of the variable are indicated, with $F$ and p-values of the ANOVA. Significantly different groups in the REGW-Q comparisons are added as dashed lines on the figures.

\begin{tabular}{|c|c|c|c|c|c|c|c|c|}
\hline Figure & Variable & Experiment & $\mathbf{N}$ & Mean & S.e. & $\mathbf{F}$ & $\operatorname{Pr}>\mathrm{F}$ & Adjusted $\mathrm{R}^{2}$ \\
\hline Fig. $1 \mathrm{~A}$ & $\mathrm{pH}$ & Mar16 & 238 & 4.9 & 1.0 & 255.2 & $<<0,0001$ & 0.95 \\
\hline Fig. $1 \mathrm{~A}$ & Eh & Mar16 & 238 & 474.6 & 69.6 & 114.7 & $<0,0001$ & 0.90 \\
\hline Fig. $1 \mathrm{~A}$ & $\mathrm{Eh}(\mathrm{pH} 7)$ & Mar16 & 238 & 352.5 & 78.0 & 97.5 & $;<0,0001$ & 0.89 \\
\hline Fig. 1B & $\mathrm{pH}$ & Jan15 & 21 & 5.0 & 0.5 & 53.6 & $<0,0001$ & 0.94 \\
\hline Fig. 1B & $\mathrm{pH}$ & Feb15 & 24 & 4.7 & 1.0 & 91.2 & $<0,0001$ & 0.96 \\
\hline Fig. 1B & $\mathrm{pH}$ & Jul15 & 60 & 4.9 & 0.8 & 171.3 & $<<0,0001$ & 0.95 \\
\hline Fig. 1B & Eh & Jan15 & 21 & 501.5 & 66.3 & 76.7 & $<0,0001$ & 0.96 \\
\hline Fig. 1B & Eh & Feb15 & 24 & 518.8 & 73.2 & 58.3 & $<<0,0001$ & 0.95 \\
\hline Fig. 1B & Eh & Jul15 & 60 & 482.1 & 76.7 & 242.4 & $t<0,0001$ & 0.96 \\
\hline Fig. 1B & $\mathrm{Eh}(\mathrm{pH} 7)$ & Jan15 & 21 & 378.9 & 63.5 & 105.9 & $<0,0001$ & 0.97 \\
\hline Fig. 1B & $\mathrm{Eh}(\mathrm{pH} 7)$ & Feb15 & 24 & 383.6 & 60.2 & 35.4 & $t<0,0001$ & 0.91 \\
\hline Fig. 1B & $\mathrm{Eh}(\mathrm{pH} 7)$ & Jul15 & 60 & 355.8 & 58.2 & 124.7 & $<0,0001$ & 0.93 \\
\hline Fig. 3 & Dia Ab177 & Jan15 & 78 & 22.6 & 5.5 & 190.9 & $<0,0001$ & 0.97 \\
\hline Fig. 3 & Dia Ab177 & Feb15 & 78 & 24.9 & 11.4 & 772.9 & $<0,0001$ & 0.99 \\
\hline Fig. 3 & Dia Ab177 & Jul15 & 234 & 27.1 & 14.7 & 297.4 & $t<0,0001$ & 0.94 \\
\hline Fig. 3 & Dia LbB3.5 & Jan15 & 78 & 15.1 & 6.6 & 291.3 & $3<0,0001$ & 0.98 \\
\hline Fig. 3 & Dia LbB3.5 & Feb15 & 78 & 18.2 & 10.4 & 297.6 & $<<0,0001$ & 0.98 \\
\hline Fig. 3 & Dia LbB3.5 & Jul15 & 234 & 17.6 & 15.0 & 277.9 & $<0,0001$ & 0.93 \\
\hline Fig. 3 & Dia LmJN2 & Jan15 & 78 & 11.5 & 6.9 & 45.3 & $3<0,0001$ & 0.87 \\
\hline Fig. 3 & Dia LmJN2 & Feb15 & 78 & 22.7 & 11.7 & 74.2 & $<0,0001$ & 0.92 \\
\hline Fig. 3 & Dia LmJN2 & Jul15 & 234 & 28.3 & 10.4 & 292.6 & $<<0,0001$ & 0.94 \\
\hline Fig. 3 & Dia Sm713 & Jan15 & 78 & 26.7 & 8.3 & 15.0 & $<0,0001$ & 0.69 \\
\hline Fig. 3 & Dia Sm713 & Feb15 & 78 & 17.9 & 15.1 & 100.9 & $<0,0001$ & 0.94 \\
\hline Fig. 3 & Dia Sm713 & Jul15 & 234 & 42.3 & 13.7 & 236.0 & $<0,0001$ & 0.92 \\
\hline Fig. 3 & Dia Ss448 & Jan15 & 78 & 19.4 & 9.1 & 44.3 & $3<0,0001$ & 0.87 \\
\hline Fig. 3 & Dia Ss448 & Feb15 & 78 & 8.8 & 9.4 & 50.4 & $t<0,0001$ & 0.89 \\
\hline Fig. 3 & Dia Ss448 & Jul15 & 234 & 12.4 & 13.8 & 316.8 & $3<0,0001$ & 0.94 \\
\hline Fig. 3 & Dia Vd586 & Jan15 & 78 & 20.9 & 5.7 & 171.9 & $<0,0001$ & 0.96 \\
\hline Fig. 3 & Dia Vd586 & Feb15 & 78 & 16.7 & 5.7 & 75.3 & $3<0,0001$ & 0.92 \\
\hline Fig. 3 & Dia Vd586 & Jul15 & 234 & 18.9 & 7.1 & 156.7 & $<0,0001$ & 0.89 \\
\hline Fig. 3 & Dia MoBN1162 & Jan16 & 80 & 63.0 & 9.9 & 1999.1 & $<0,0001$ & 1.00 \\
\hline Fig. 3 & Dia MoBN1168 & Mar16 & 70 & 63.5 & 9.7 & 119.0 & $<0,0001$ & 0.97 \\
\hline
\end{tabular}

\subsection{Effect of fungi on $\mathrm{pH}$ and Eh in plant stems}

Uninfected oilseed rape stems were slightly acidic ( $\mathrm{pH}$ from 5.5 to 6.5) like malt-agar media, but more reduced (Eh from 150 to 300) as compared to malt-agar media (Eh from 500 to 600).

Analysis of deviance showed that both inoculation status and canker length were significant predictors of changes in $\mathrm{pH}$ (Table 3), though in opposite directions depending on the fungal species. $\mathrm{pH}$ was higher following fungal infection with L. maculans (Fig. 2A; Table 3) whereas $\mathrm{pH}$ was lower with S. sclerotiorum (Fig. 2E; Table 3). For both fungi, canker length had a significant effect on measured pH (Fig. 2B; Fig. 2F, Table 3). 
Table 3. Analysis of deviance on log $N$ transformed data (Type II tests). $\operatorname{Pr}\left(\chi^{2}\right)$ are $p$-values from a $\chi^{2}$ test used for significance.

\begin{tabular}{|l|l|l|l|l|l|}
\hline Model & Tested effect & Df & $\mathbf{X} 2$ & $\operatorname{Pr}(>\mathbf{X} \mathbf{2})$ & \\
\hline L. maculans $\mathrm{pH}$ & Canker & 1 & $1.28 \times 10^{2}$ & $<2 \times 10^{-16}$ & $* * *$ \\
\hline & Infection & 2 & $1.48 \times 10^{5}$ & $<2 \times 10^{-16}$ & $* * *$ \\
\hline & Replicate & 2 & 2.41 & 0.30 & \\
\hline L. maculans Eh & Canker & 1 & 2.51 & 0.11 & \\
\hline & Infection & 2 & $1.26 \times 10^{5}$ & $<2 \times 10^{-16}$ & $* * *$ \\
\hline & Replicate & 2 & 0.56 & 0.75 & \\
\hline S. sclerotiorum $\mathrm{pH}$ & Canker & 1 & 616.64 & $<2 \times 10^{-16}$ & $* * *$ \\
\hline & Infection & 2 & 32608.66 & $<2 \times 10^{-16}$ & $* * *$ \\
\hline & Replicate & 1 & 3.01 & 0.08 &. \\
\hline & & & & & \\
\hline S. sclerotiorum Eh & Canker & 1 & $1.75 \times 10^{2}$ & $<2 \times 10^{-16}$ & $* * *$ \\
\hline & Infection & 2 & $1.30 \times 10^{5}$ & $<2 \times 10^{-16}$ & $* * *$ \\
\hline & Replicate & 1 & 2.65 & 0.10 & \\
\hline
\end{tabular}

*** denote significance, with probability below the 0.05 level of risk. 
Fig. 2. Effect of fungal growth on the $p H$ and Eh of oilseed rape stems for L. maculans (A to D) and S. sclerotiorum (E to H). Boxplot of $\mathrm{pH}(A$ and $E)$ or $E$ h $(C$ and $G)$ at stem base on plants uninfected or infected for the three vs. two replicates $(R 1, R 2, R 3)$. Measured $p H(B$ and $F)$ or Eh $(D$ and $H)$ in stems depending on canker length, symbols differ for the three vs. two sets of plants.
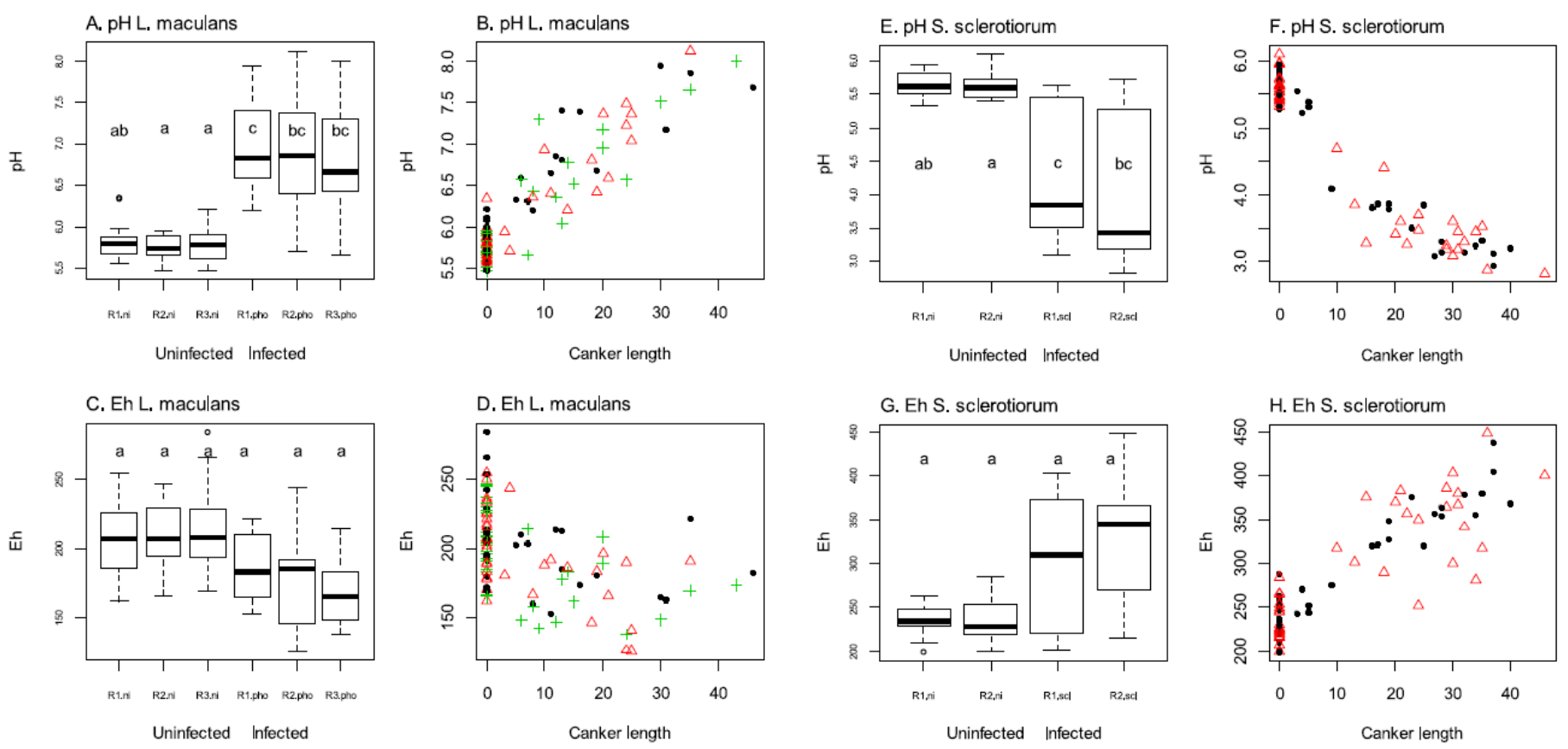

Analysis of deviance showed that inoculation status was a significant predictor of changes in Eh (Table 3), again in opposite directions depending on the fungal species. Eh was lower following fungal infection with L. maculans though differences between groups were not significant (Fig. 2C; Table 3) whereas Eh was higher with S. sclerotiorum (Fig. 2G; Table 3). The effect of canker length on Eh was significant for S. sclerotiorum (Fig. 2H; Table 3) but not for L. maculans (Fig. 2D; Table 3).

\subsection{Effect of $p H$ and $E h$ in the agar media on fungal growth}

The addition of electrolyzed water products to malt-agar media extended the $\mathrm{pH}$ and Eh range on both sides of the native medium (Fig. 3 right panel) and on both sides of the range from 5.5 to 6.5 observed in oilseed rape stems (Fig. 2). The Eh-pH couples obtained did align on the theoretical correlation between Eh and $\mathrm{pH}$, with a negative slope of $-59 \mathrm{mV} / \mathrm{pH}$ unit. The native rice flour medium was $\mathrm{pH} 6.5$, higher than the native malt medium at $\mathrm{pH} 4.5-5$; and it was more reduced, with Eh $400 \mathrm{mV}$ compared to Eh 550-600 mV. The addition of Hydrogen peroxide, and Hydroquinone to rice flour media allowed variation in Eh (from 300 to $500 \mathrm{mV}$ ), but less variation in $\mathrm{pH}(6-7)$ (Fig. 3 right panel). 
Fig. 3. Mean colony growth of 6 fungi (each on one row of the figure) depending on pH (left panel), Eh (central panel), and the combination of $\mathrm{pH}$ and Eh (right panel) in the media prepared in three independent experiments Jan2015 (diamonds) Feb2015 (triangles) and Jul2015 (squares). Polynomial regression over all pH and Eh data is indicated. Each point is mean of 2 measures on 3 colonies (Jan2015, Feb2015) and 9 colonies (Jul2015). Note that $\mathrm{pH}$ X-axis scale is different for M. oryzae. Line in the right panel shows the theoretical relationship between $\mathrm{pH}$ and $\mathrm{Eh}$, with a slope of $-59 \mathrm{mV} / \mathrm{pH}$ unit according to the equation of Nernst.
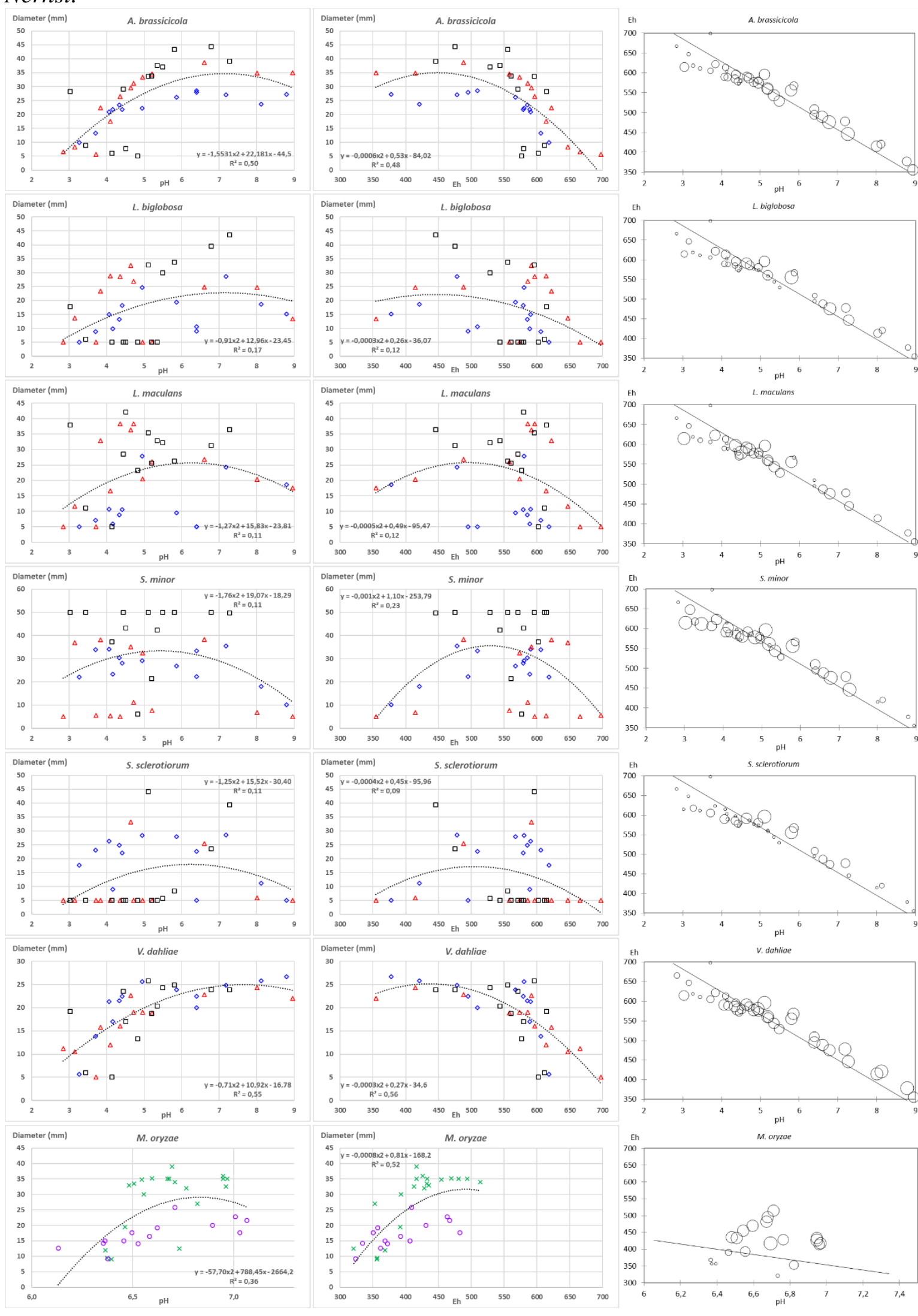
For the six fungi tested, mean colony diameter varied on the range of 13 malt-agar media (Fig. 3; Table 2). Further, the colony appearance of all fungi, the pattern of sclerote production for S. minor and $S$. sclerotiorum, the production of yellow pigments for Leptosphaeria biglobosa varied in a manner congruent between replicated dishes in independent preparations (Supplementary information Fig. S3). In most cases, the fungal response to the modified media was quantitative with a decrease in colony diameter. However, in a few occasions the response was also qualitative, with the fungus either remaining on the transferred agar plug for some of the dishes, or starting to grow on some other dishes (Fig S3). The growth of Alternaria brassicicola, L. biglobosa and Verticillium dahliae was better at higher $\mathrm{pH} /$ lower Eh than at lower $\mathrm{pH} / \mathrm{higher}$ Eh, with optimum $\mathrm{pH}$ around 7-8 and optimum Eh around $450 \mathrm{mV}$ (Fig. 3). The growth of L. maculans was reduced on both sides of the range tested, with optimum $\mathrm{pH}$ around 6 and optimum Eh around $500 \mathrm{mV}$. The optimum were lower for $S$. minor with $\mathrm{pH} 5-6$, and Eh below $400 \mathrm{mV}$ than for S. sclerotiorum with $\mathrm{pH}$ around 6 and Eh around $450 \mathrm{mV}$.

In the Sep15 experiment, the addition up to $500 \mathrm{ppm}$ of $\mathrm{NaCl}$ to the malt-agar media influenced Eh and $\mathrm{pH}$ (Fig. S1A). All media were more reduced (Eh from 480 to $495 \mathrm{mV}$ ) than the native one $(\mathrm{Eh}=505 \mathrm{mV})$. Compared to the native one $(\mathrm{pH}=5.6)$, the $\mathrm{pH}$ was decreased $(5.45$ and 5.5) only for the addition of 100 and $500 \mathrm{ppm}$ of $\mathrm{NaCl}$. $\mathrm{NaCl}$ did not alter the colony growth of the five fungi S. minor, L. maculans, L. biglobosa, A. brassicicola and V. dahliae. The colony growth of S. sclerotiorum differed among media, though the only growth different from the native medium N0 was with $250 \mathrm{ppm}$ of $\mathrm{NaCl}$ (Fig. S1B).

\section{Discussion}

Congruent results in independent experiments indicate that the protocol developed for measurements in soil (Husson et al., 2016) can also be used for measurement in agar media. The growth of all fungi tested significantly altered either Eh, pH or both in agar media (Fig. 1A, B). This effect was consistently observed among replicates within each experiment, and among independent experiments. Changes in $\mathrm{pH}$ were congruent with previous literature for fungi notorious to acidify (e.g. Sclerotinia) or alkalinize (e.g. Magnaporthe, Botrytis) the media (Landraud et al., 2013, Liu et al., 2017, Vylkova, 2017). Changes in Eh were congruent with previous literature, with various aerobes strongly reducing the culture media, due to the consumption of oxygen (Rabotnova and Schwartz, 1962). Interestingly, the joint characterization of $\mathrm{pH}$ and Eh provides discrimination among species not discriminated by the $\mathrm{pH}$ alone. Measuring Eh reveals that even the species not modifying $\mathrm{pH}$ can have an impact on the surrounding environment. For plant crop species, realizing that the plant pathogens might interfere with the plant oxido-reduction status (De Gara et al., 2003, Williams et al., 2011, Kabbage et al., 2015) and thus that maintaining redox homeostasis might come at a cost for an infected plant, opens another direction of investigation to better understand pathogen damage to plants.

Congruent results with two different fungi indicate that the protocol developed for measurements in soil (Husson et al., 2016) can also be used for measurement in infected plants. In infected plant stems, $\mathrm{pH}$ was significantly altered, in opposite directions for L. maculans and S. sclerotiorum (Fig. 2). The observed alkalinization or acidification correlates with canker length. The direction of this change is congruent with changes observed on agar media (Fig. 1A, B). As the measurements in plants result both from fungal growth and from the plant reaction to infection, follow-up studies are needed to disentangle the respective roles of fungal mycelium, of fungal metabolism and of plant reaction (Casadevall and Pirofski, 2003). It is known that disease severity depends on plant organ age (Kranz, 1990, Benada, 2017, Torres et al., 2017) and some pathogens are only able to infect specific of plant organs. Being able to perform direct measurements on a small amount of plant material will allow detailed studies to test whether the change is only local or systemic in the plant.

On modified agar media, we observed that most fungi could grow over a broad pH range (Fig. 3) which is congruent with previous literature. Among the species tested, $\mathrm{pH}$ optimum was the highest for V. dahliae (Fig. 3) around $\mathrm{pH} 7$ to 8. This fungus is able to grow hyphae in soil, which $\mathrm{pH}$ is less acidic that plant environment. For A. brassicicola, optimal pH between 5.5 and 7.5 was reported (Dinh et al., 2016). This is congruent with our results (Fig. 3). In S. sclerotiorum, oxalic acid is required for plant infection (Wang et al., 2016) and mediates the host redox environment (Williams et al., 2011, Kabbage et al., 2015). We observed 
both that S. sclerotiorum strongly acidifies the medium (Fig. 1A, B) and that fungal growth is reduced at $\mathrm{pH}$ below 6, being stopped at $\mathrm{pH} 3$ (Fig. 3). Thus, the modification of the surrounding environment by the fungus might retroact on the fungus.

As compared to $\mathrm{pH}$, information on the effect of redox potential on fungal growth is scarce. In our experiment, beside data for colony diameter (Fig. 3) it was striking to see, that colony aspect was repeatedly and thoroughly modified (Fig. S3). In our experiment, the amount and pattern of sclerote production was strongly affected in S. minor, and to a lesser extent in S. sclerotiorum (Fig. S3). This is congruent with previous reports that sclerotial differentiation may be induced by reactive oxygen species (Georgiou and Petropoulou, 2001). We noticed that sclerote and pigment production, mycelium color and aspect were affected and further studies are needed to quantify these changes.

Eh and $\mathrm{pH}$ are inversely correlated, and this process can be explained because oxidation of two molecules of water (losing 4 electrons) produces $\mathrm{O}_{2}$ and $4 \mathrm{H}^{+}$, thus leading to acidification. From the Nernst equation, it can be calculated that the theoretical slope of this correlation is a $59 \mathrm{mV}$ decrease in Eh for one unit increase in $\mathrm{pH}$ at $25^{\circ} \mathrm{C}$ (Husson et al., 2018). In our experiment, we observed that without biological activity, all the agar media prepared align on this theoretical line (Fig. 3 right panel). In contrast, the final measurements in agar media after fungal growth do not match this theoretical correlation line (Fig. 1A, B). Rabotnova and Schwartz (1962) postulate that the observed reduction of the media is due to the consumption of dissolved oxygen. Further studies on media with different compositions are needed to investigate whether the final values depend on the consumption/release of different nutrient and compounds by the fungi, or if final values are stable, thus indicating regulation.

\section{Conclusion}

As a conclusion, our series of experiments indicate that the procedure published for Eh and $\mathrm{pH}$ in soil (Husson et al., 2016, Husson et al., 2018) can be extended for measurement in agar media and in infected plants. Further, the joint characterization of both parameters opens the way to a more precise understanding of the impact of fungi on their environment, and conversely, of the environment on fungal growth. This could have applications at the interface between plant pathology, microbial ecology and plant physiology. Indeed, disease severity interacts with organ age (Kranz, 1990) or senescence (Häffner et al., 2015) or nutrient status (Veresoglou et al., 2013). Contrasting disease outcomes have been described when different fungi grow on co-infected plants (Lamichhane and Venturi, 2015, Abdullah et al., 2017). However, a comprehensive understanding of interactions or combinations of stresses is lacking (Nejat and Mantri, 2017, Pandey et al., 2017). Publications at the molecular level are congruent with an implication of oxido-reduction processes (Shigeoka and Maruta, 2014, Nosek et al., 2015, Marschall and Tudzynski, 2016, Choudhury et al., 2017, Noctor et al., 2017). These lines of evidence points to $\mathrm{pH}$ and Eh as key signaling factors and hub of stress in the plant-pathogen interactions. The availability of methods for measurement opens the prospect to study combinations of stresses, either on agar media or in plants, and get an understanding of the involvement of $\mathrm{pH}$ and Eh modifications in these interactions.

\section{Acknowledgments}

We thank the two anonymous reviewers for helping us improving the manuscript. All authors declare the absence of conflicts of interest. This work benefited from the financial support of INRA - the French National Institute for Agronomical Research, with no implication in conducting the study.

Additional Supporting Information may be found in the online version of this article:

Fig. S1 Effect of $\mathrm{NaCl}$ in the Sep15 experiment A. on the $\mathrm{pH}$ and $\mathrm{Eh}$ of the uninoculated malt-agar media; B. on the growth S. sclerotiorum colonies. Uninoculated medium (Panel A) was native (filled symbol) or with addition of $\mathrm{NaCl}$ up to $500 \mathrm{ppm}$ (empty symbols). Dashed lines correspond to significantly different groups for $\mathrm{pH}$ (horizontal) and Eh (vertical) in the REGWQ analysis. In Panel B, size of circles correspond to colony diameter. Letters correspond to significantly different groups in the REGWQ analysis. 
Fig. S2 Measurement of Eh on plant stems. The plant material grinded with a mortar and pestle was placed in the barrel of a $5 \mathrm{ml}$ syringe from which the nozzle had been cut. In the $9 \mathrm{~cm}$ Petri dish, the filter paper was moistened with $0.1 \mathrm{M} \mathrm{KCl}$ solution. The $\mathrm{Ag} / \mathrm{AgCl}$ reference electrode was placed standing, with tip touching the moist filter paper. The Pt plate electrode was placed in the plant material in the syringe, then the syringe barrel was placed standing with tip touching the moist filter paper.

Fig. S3 Colony aspect at the end of the Jul15 experiment on native and altered agar media for six fungi. Each panel correspond to a single strain transferred the same day and grown in the same room, with the 13 media in columns and replicates in lines. Within the experiment, lines 1 to $3 ; 4$ to $6 ; 7$ to 9 correspond to the three independent replicates (i.e. is preparation in independent bottles, and inoculation with agar plugs from a different source plate). Within upper, central and lower groups, dishes were prepared from the same bottles for all fungi. Mean $\mathrm{pH}$ and Eh values measured on aliquots corresponding to the 13 columns are given below. Note: colony diameter had been assessed earlier than the picture for fast growing fungi. Dishes are $5 \mathrm{~cm}$ in diameter.

\section{References}

Abdullah, A.S., Moffat, C.S., Lopez-Ruiz, F.J., Gibberd, M.R., Hamblin, J., Zerihun, A., 2017. Host-multipathogen warfare: pathogen interactions in co-infected plants. Front. Plant Sci. 8. 1806.

Addinsoft, 2016. XLSTAT: Data analysis and statistics with Microsoft Excel. Paris, France.

Bekker, T.F., Kaiser, C., Labuschagne, N., 2009. The antifungal activity of potassium silicate and the role of $\mathrm{pH}$ against selected plant pathogenic fungi in vitro. South African Journal of Plant Soil. 26. 55-56.

Benada, J., 2017. Measurement of redox potential and $\mathrm{pH}$ in plants and their function in the mechanism of plant resistance and in plant physiology. International Journal of Advanced Research in Electrical, Electronics and Instrumentation Engineering. 6. 1111-1116. DOI:10.15662/IJAREEIE.2015.0501001

Braunsdorf, C., Mailänder-Sánchez, D., Schaller, M., 2016. Fungal sensing of host environment. Cell. Microbiol. 18. 1188-1200.

Casadevall, A., Pirofski, L.A., 2003. The damage-response framework of microbial pathogenesis. Nature Rev. Microbiol. 1. 17-24.

Choudhury, F.K., Rivero, R.M., Blumwald, E., Mittler, R., (2017) Reactive oxygen species, abiotic stress and stress combination. The Plant Journal. 90. 856-867.

Daval, S., Lebreton, L., Gracianne, C., Guillerm-Erckelboudt, A.Y., Boutin, M., Marchi, M., Gazengel, K., Sarniguet, A., 2013. Strain-specific variation in a soilborne phytopathogenic fungus for the expression of genes involved in $\mathrm{pH}$ signal transduction pathway, pathogenesis and saprophytic survival in response to environmental pH changes. Fung. Genet. Biol. 61. 80-9.

De Gara, L., de Pinto, M.C., Tommasi, F., 2003. The antioxidant systems vis-à-vis reactive oxygen species during plant-pathogen interaction. Plant Physiol. Bioch. 41. 863-870.

Dietz, K.J., Mittler, R., Noctor, G., 2016. Recent progress in understanding the role of reactive oxygen species in plant cell signaling. Plant Physiol. 171. 1535-1539.

Dinh, V.T., Somasekhara, Y.M., Shivaprakash, M.K., 2016. Cultural and physiological studies of Alternaria brassicicola (Schw.) Wiltshire of cabbage (Brassica oleracea var. capitata L.). Mysore J. Agric. Sci. 50. 529-534.

Foyer, C.H., Noctor, G., 2005. Redox homeostasis and antioxidant signaling: a metabolic interface between stress perception and physiological responses. The Plant Cell. 17. 1866-1875.

Foyer, C.H., Noctor, G., 2016. Stress-triggered redox signalling: what's in prospect? Plant, Cell Envir. 39. 951-964.

Georgiou, C.D., Petropoulou, K.P., 2001. Effect of the antioxidant ascorbic acid on sclerotial differentiation in Rhizoctonia solani. Plant Pathol. 50. 594-600.

Häffner, E., Konietzki, S., Diederichsen, E., 2015. Keeping control: the role of senescence and development in plant pathogenesis and defence. Plants. 4. 449-488.

Howell, D.C., 2009. Statistical methods for psychology. Cengage Learning. Wadsworth 
Husson, O., 2013. Redox potential (Eh) and $\mathrm{pH}$ as drivers of soil/plant/microorganism systems: a transdisciplinary overview pointing to integrative opportunities for agronomy. Plant and Soil. 362. 389417.

Husson, O., Brunet, A., Babre, D., Charpentier, H., Durand, M., Sarthou, J.P., 2018. Conservation Agriculture systems alter the electrical characteristics (Eh, pH and EC) of four soil types in France. Soil and Tillage Research.176. 57-68.

Husson, O., Husson, B., Brunet, A., Babre, D., Alary, K., Sarthou, J.P., Charpentier, H., Durand, M., Benada, J., Henry, M., 2016. Practical improvements in soil redox potential (Eh) measurement for characterisation of soil properties. Application for comparison of conventional and conservation agriculture cropping systems. Analytica Chimica Acta. 906. 98-109.

Jwa, N.S., Hwang, B.K., 2017. Convergent evolution of pathogen effectors toward reactive oxygen species signaling networks in plants. Front. Plant Sci. 8. 1687.

Kabbage, M., Yarden, O., Dickman, M.B., 2015. Pathogenic attributes of Sclerotinia sclerotiorum: Switching from a biotrophic to necrotrophic lifestyle. Plant Sci. 233. 53-60.

Kranz, J., 1990. Fungal disease in multispecies plant communities. New Phytol. 116. 383-405.

Lamichhane, J.R., Venturi, V., 2015. Synergisms between microbial pathogens in plant disease complexes: a growing trend. Front. Plant Sci. 6. 385.

Landraud, P., Chuzeville, S., Billon-Grande, G., Poussereau, N., Bruel, C., 2013. Adaptation to pH and role of PacC in the rice blast fungus Magnaporthe oryzae. PLoS ONE 8(7): e69236.

Lebreton L., Daval S., Guillerm-Erckelboudt, A.Y., Gracianne, C., Gazengel, K., Sarniguet, A., 2014. Sensitivity to $\mathrm{pH}$ and ability to modify ambient $\mathrm{pH}$ of the take-all fungus Gaeumannomyces graminis var. tritici. Plant Pathol. 63. 117-128.

Lehmann, S., Serrano, M., L'Haridon, F., Tjamos, S.E., Metraux, J.P., 2015. Reactive oxygen species and plant resistance to fungal pathogens. Phytochemistry. 112. 54-62.

Liu, J., Zhang, Y., Meng, Q., Shi, F., Ma, L., Li, Y., 2017. Physiological and biochemical responses in sunflower leaves infected by Sclerotinia sclerotiorum. Physiol. Mol. Plant Pathol. 100. 41-48.

Lo Presti, L., Lanver, D., Schweizer, G., Tanaka, S., Liang, L., Tollot, M., Zuccaro, A, Reissmann, S., Kahmann, R., 2015. Fungal effectors and plant susceptibility. Ann. Rev. Plant Biol. 66. 513-545.

Marschall, R., Tudzynski, P., 2016. Reactive oxygen species in development and infection processes. Seminars in Cell and Develop. Biol. 57. 138-146.

Nejat, N., Mantri, N., 2017. Plant immune system: crosstalk between responses to biotic and abiotic stresses the missing link in understanding plant defence. Current Issues in Mol. Biol. 23. 1-16.

Noctor, G., Reichheld, J.P., Foyer, C.H., 2017. ROS-related redox regulation and signaling in plants. Seminars in Cell and Develop. Biol. 80. 3-12.

Nosek, M, Kornaś, A., Kuźniak, E, Miszalski, Z., 2015. Plastoquinone redox state modifies plant response to pathogen. Plant Physiol. Bioch. 96. 163-170.

Pandey, P., Irulappan, V., Bagavathiannan, M.V., Senthil-Kumar, M., 2017. Impact of combined abiotic and biotic stresses on plant growth and avenues for crop improvement by exploiting physio-morphological traits. Front. Plant Sci. 8. 537.

R Core Team, 2013. R; a language and environment for statistical computing. R Foundation for Statistical Computing, Vienna, Austria. URL http://www.R-project.org/.

Rabotnova, I.L., Schwartz, W., 1962. The importance of physical-chemical factors (pH and rH2) for the life activity of microorganisms. Microbiology. VEB Gustav Fisher Verlag, Jena.

Shigeoka, S., Maruta, T., 2014. Cellular redox regulation, signalling, and stress response in plants. Biosci. Biotechnol. and Bioch. 78. 1457-70.

Smiley, R.W., 1974. Take-all of wheat as influenced by organic amendments and nitrogen fertilizer. Phytopathol. 64. 822-825.

Stiles, C.M., Murray, T.D., 1996. Infection of field-grown winter wheat by Cephalosporium gramineum and the effect of soil pH. Phytopathol. 86. 177-183.

Torres, D.P., Proels, R.K., Schempp, H., 2017. Silencing of RBOHF2 causes leaf age-dependent accelerated senescence, salicylic acid accumulation, and powdery mildew resistance in barley. MPMI. 30. 906-918. 
van der Does, H.C., Rep, M., 2017. Adaptation to the host environment by plant-pathogenic fungi. Ann. Rev. Phytopathol. 55. 427-450.

Veresoglou, S.D., Barto, E.K., Menexes, G. Rillig, M.C., 2013. Fertilization affects severity of disease caused by fungal plant pathogens. Plant Pathol. 62. 961-969.

Vylkova, S., 2017. Environmental pH modulation by pathogenic fungi as a strategy to conquer the host. PLoS Pathogens. 13(2): e1006149.

Williams, B., Kabbage, M., Kim, H.J., Britt, R., Dickman, M.B., 2011. Tipping the balance: Sclerotinia sclerotiorum secreted oxalic acid suppresses host defenses by manipulating the host redox environment. PLoS Pathogens. 7. Doi=10.1371/journal.ppat.1002107.

Zeilinger, S., Gupta, V.K., Dahms, T.E.S., Silva, R.N., Singh, H.B., Upadhyay, R.S., Vieira Gomes, E., Tsui, C.K.M., Nayak, S.C., 2016. Friends or foes? Emerging insights from fungal interactions with plants FEMS Microbiol. Rev. 40. 182-207. 